\title{
Residual Entropy of Linear Polymers
}

\author{
H. N. V. Temperley
}

\begin{abstract}
The question whether it is possible to assign a finite entropy to a polymer, bearing in mind the fact that it may not be a well-defined substance in thermodynamic equilibrium, is considered. It is shown that the entropy of chain configurations is the only contribution that will be important at low temperatures. Existing knowledge of the thermodynamics of hydrocarbons enables one to arrive at a generalization of the well-known "flexible chain" model of a polymer molecule. The new model is in good accord with experimental facts on the specific heat of polymers, and enables a finite entropy to be defined and to be reasonably accurately calculated. These findings are then compared with certain experimental results throwing light on the length of the "effective segment" of polymer chains of various types, this quantity being defined in the text. The experimental facts come from studies of polymer solutions, solid polymers, solid and liquid paraffins, and related compounds, and are remarkably consistent with the theoretical expectations. A few apparent inconsistencies and a few instances of alternative possible interpretations for the same facts are noted.
\end{abstract}

\section{Introduction}

In attempting to predict heats of polymerization from thermochemical data on long-chain hydrocarbons and the monomer, it is not unusual to meet discrepancies between calculated and observed values of the order of 1 to $10 \mathrm{kcal} / \mathrm{mole}$, Flory [5, ch. 6; p. 254]. ${ }^{1}$ After a careful survey of the evidence, Flory concluded that: "The pattern presented by heats of polymerization of various monomers appears to be very well explained by two dominant factors: elimination of resonance energy of conjugation, and steric interaction between substituents." In other words, the discrepancies can be explained purely on chemical considerations; the necessary readjustments of electronic configurations associated with the joining together of monomers can be much more important in some types of chain than in other types.

However, it is sometimes pointed out that errors might be caused by treating a polymer as a welldefined chemical substance in true thermodynamic equilibrium. This question may also be of interest in the study of other polymer reactions such as degradation, in which the main chain is broken up into fragments or greatly modified. (For reactions in which the main chain remains practically intact, errors in calculations of the configurational entropy will tend to balance out, since they affect both reactants and products.) The present study was undertaken in an effort to estimate numerical values for the residual entropy of a typical linear polymer, bearing in mind the four facts: first, that it is inevitably a mixture of chains of many different lengths; second, that because of "freedom at the joints" any one chain may assume a wide variety of configurations in space; third, that in a copolymer, the number of ways in which two sets of monomers can be lined up to form a chain can be enormously great; finally, that at least some of any polymer is in an amorphous or disordered state. It is known that crystallization of a polymer is never complete, and some polymers cannot crystallize at all. Moreover, polymers undergo a "glass-like" transition at

\footnotetext{
${ }_{1}^{1}$ Figures in brackets indicate the literature references at the end of this paper.
}

temperatures at which thermal agitation is still considerable, so that some "frozen-in disorder" is to be expected. We are interpreting the glass-like transition as being essentially the same as that of an inorganic glass, certain degrees of freedom being put out of action as a result of a rapid rise in their time-constants with falling temperature. Some workers have claimed that there is a true phase transition, but a discussion of this point is hardly relevant in the present context. See Kauzmann [12], Powell and Eyring [25], Boyer and Spencer [2]. It might seem a hopeless task to identify the separate contributions to the residual entropy associated with each of the four factors listed above, but the task is very much simplified when it is realized that contributions of less than about $R$ per mole of monomer are unlikely to be of practical interest. At ordinary temperatures such an entropy calls for a correction to the free energy of $1 / 2$ to 1 kcal/mole. It can very quickly be shown that the contributions due to 2 of the 4 causes listed above are negligibly small, while the entropy associated with copolymerization can probably be estimated fairly accurately in any given case. We are thus left only with the problem of estimating the number of different configurations in which a real molecule of polymer type can exist in a solid or liquid. It is this study that forms the subject of the present paper, but the other three possible contributions to the residual entropy will be considered first.

\section{Contributions From Causes Other Than Chain Configurations}

\subsection{Communal Entropy}

We shall consider the effect of chain configurations in detail, but must first discuss a different effect, namely, that in a crystalline solid it is sufficient to think of each molecule as never straying very far from a given lattice point, but in a liquid, gas, or amorphous solid, it may be necessary to allow a typical molecule "effective access" to an appreciable function of the whole volume. For 
ordinary liquids this leads to the tiresome "communal entropy" problem that is still unsolved. For polymers, however, it is readily shown that as a consequence of the great size of the molecules, any contribution to the entropy resulting from the bodily transport of a whole molecule around the assembly is quite negligible compared with the contributions due to configuration effects. Physically, the coordinates of the center of gravity of a chain represent just three out of the hundreds of coordinates that would have to be known in order to specify the positions of all the elements of a chain. More quantitatively, if we permitted the center of gravity of a chain to wander over the entire volume $V$ of an assembly of $N$ chains instead of restricting it to its own "share" $V / N$ of that volume, the gain in entropy would be $k \ln N$ per chain or $N k \ln N$ for the assembly. However, this count includes changes of configuration that amount to exchanging the positions of two or more similar chains, which does not lead to a physically distinguishable configuration. The number of permutations of $N$ chains among themselves is $N$ !, so the proper contribution to the entropy, if the chains are all alike, is not more than

$$
N k \ln N-k \ln (N !) \approx N k
$$

or $k$ per chain. It is readily shown that any interaction between the chains, whether it is an attraction or repulsion, can only result in some departure from complete randomness, thus making the contribution to the entropy smaller. Even if one argued that two polymer chains could be made physically distinguishable, e. g., by some slight differences in the substituents, so that it became physically proper to drop the $N$ ! term, the communal entropy would still only be $k \ln N$ per chain. For an assembly of ordinary size $N \approx 10^{20}$ this would amount to something of the order of $50 \mathrm{k}$ per chain of perhaps 1,000 monomers, whereas, as already shown above, we are looking for effects of the order of $k$ per monomer molecule. Moreover, a preparation in which the number of distinguishable species of linear molecules approached the number of molecules present could only be described as an extremely bad one chemically. We can conclude, therefore, that the contribution due to communal entropy is always negligible. Besides effects associated with the translation of entire molecules, one also should consider possible contributions due to the orientation of the molecule as a whole, the relative positions of all the segments being held fixed. The number of such possible orientations is certainly no greater than the number of physically distinguishable ways in which one single segment of the molecule, for example, the end segment, can be oriented relative to its immediate surroundings. The order or magnitude of this number is readily estimated from entropy data on ordinary liquids and solids.

The total entropy of an ordinary liquid or solid at ordinary temperatures is of the order of a few times $R$. Even if it were assumed that all of this entropy is associated with the communal effect, together with possible orientations and "rattling. around" of a molecule of liquid relative to its immediate surroundings, this would still mean that the number of physically distinguishable states of a typical molecule effectively excited at ordinary temperatures is no more than $e^{3}$ to $e^{5}$ (about 10 to 100). From this it can be quite safely concluded that the translations and orientations of entire molecules of polymer of fixed configurations can contribute no more than 3 to 5 times $k$ per polymer chain.

\subsection{Differing Molecular Weights}

It is well known that any linear polymer preparation contains molecules of all possible lengths from the monomer upwards, and it is proper to regard chains of two different lengths as "physically distinguishable." Since chains of more than a few thousand units long seldom occur in practice, the number of species effectively present will be small compared with the total number of chains. Consequently, any contribution to the entropy from this cause should be small. For branched as distinct from nearly linear polymers this argument does not hold. The number of paraffin isomers $\left(\mathrm{C}_{n} \mathrm{H}_{2 n+2}\right)$ is known, G. Polya [24] to be of the order of $2.8^{n}$, so the number of possible species of branched polymer with $n=100$ to 1,000 is much greater than the number of molecules present in an ordinary assembly, in which case the omission of the $N$ ! term in the communal entropy would be correct. Even so, as shown earlier, this still leads to an upper limit that is too small to be of significance in practice.

The following argument from Beckett (private communication) enables us to set a more stringent upper limit in the linear case. We may suppose that the chain lengths are distributed about the mean value on the basis of the so-called "most probable" law of Flory [5, ch. 8, p. 319]. This may be regarded as the "worst possible" case, leading to the highest entropy of all possible distribution laws. It is equivalent to the assumption that chains of all lengths are present in proportions corresponding to thermodynamic equilibrium at the polymerization temperature. By certain methods of preparation the much narrower Poisson-type distribution can be obtained, or, by fractionation in solution, a very narrow spread can also be obtained. The entropy associated with a completely random mixture of chains of different species, where $N_{x}$ is the number of " $\mathrm{x}$-mers" is

$S_{0}=k \ln \left\{N ! / \Pi_{x} N_{n} !\right\}$ with $\sum N_{x}=N, \sum_{x} x N_{x}=M$

where $\mathrm{N}$ is the total number of chains and $M$ the total number of monomer units making up the assembly. Then the law of the most probable distribution is, Flory [5, p. 319]

$$
N_{x}=N(1-p) p^{x-1}
$$


with

$$
N=M(1-p)
$$

as the equation determining $p$. Substituting from (2) and (3) into (1), approximating to the factorials by means of Stirling's theorem, we find, after performing the summations called for by (1),

$$
S_{0} \approx-M[(1-p) \ln (1-p)+p \ln p]
$$

where $p$ is related to the average length of a chain by (3). Thus, for a degree of polymerization resulting in a chain of an average length of $t$ units, we arrive at an entropy of $\approx k \ln t$ per chain, or $k \ln t$

\subsection{Entropy Associated with Copolymerization}

If two monomers $A$ and $B$ are present in equal numbers they may be arranged in completely random fashion along each chain, unless the chemical kinetics of the reaction are such that $A$ attaches itself easily to $B$ but only with difficulty to another $A$. For example, a condensation polymerization might be like this. In the first (random) case a residua] entropy of $k$ ln 2 per monomer can be expected; in the second the chain would be almost completely ordered and the entropy practically zero. It should be noticed here that the argument must be based on kinetic rather than equilibrium considerations because a chain, once formed, is not likely to break up again in order to assume a slightly more probable configuration. (This excludes conditions where polymer degradation is rapid.) The work is readily extended to the case where the two monomers are present in the proportions $g:(l-g)$, the corresponding maximum entropy being, per link,

$$
k(-g \ln g-[1-g] \ln [1-g]) \leq k \ln 2
$$

the question whether or not it is attained again being outside the province of thermodynamics alone.

\section{Chain Configuration Problem}

We have now shown that with the possible exception of the "copolymerization entropy," which cannot be predicted solely on the basis of equilibrium considerations, the possible other contributions to the entropy are too small to be of significance. This study of the chain configuration problem falls into a number of parts.

(a) What can be said, a priori, about a paraffin-like polymer in the light of existing knowledge of the paraffin chain and of the random-walk problem?

(b) What can be reduced from a study of solid polymers?

(c) What can be deduced from studies of polymers in solution?

(d) What can be deduced from a study of related compounds such as solid and liquid long-chain paraffins?
All of these studies have contributed something to the solution of the problem, and it has been possible to draw the following conclusions with a fair degree of certainty:

(a) For a hydrocarbon-like polymer the entropy associated with chain configurations is no more than $1 / 5 k$ per link.

(b) For a rubber-like polymer the experimental and theoretical information is less abundant and less definite, but it seems safe to say that the configuration entropy is no more than $k$ per monomer.

We shall begin by asking what would be expected for a paraffin chain without side substituents, then consider briefly the possible effects of such substitutions and the presence of double bonds in the main chain. We shall then analyze the available experimental evidence in the light of these expectations.

\subsection{A priori Expectations}

A large number of studies have been made of the expected behavior of statistical assemblies of various types of jointed chains, beginaing with the simplest possible case in which the "joints" are supposed to be entirely flexible, and in which complications due to the fact that a chain may not cross itself are ignored. For a survey of this work the reader is referred to Flory [5, ch. 10]. The idea that a long chain molecule can be represented in this way has proved extremely valuable in correlating a large number of experimental facts, but needs to be refined and generalized before it can be applied to the problem of calculating entropies.

It has been pointed out by a number of writers that the freely jointed chain model is in many ways analogous to both the Langevin model of a paramagnetic and to the perfect gas. The analogous

\begin{tabular}{|c|c|c|c|c|}
\hline Perfect gas...... & Pressure...... & Volume....... & Compressibil- & Entropy. \\
\hline $\begin{array}{l}\text { Langevin mod- } \\
\text { el. }\end{array}$ & $\begin{array}{l}\text { M a g n e t i c } \\
\text { field. }\end{array}$ & $\begin{array}{l}\text { Mean mag- } \\
\text { netic mo- }\end{array}$ & Susceptibility_- & Entropy. \\
\hline $\begin{array}{l}\text { Freely jointed } \\
\text { chain. }\end{array}$ & Tension........ & $\begin{array}{l}\text { ment. } \\
\text { Mean length } \\
\text { of chain. }\end{array}$ & $\begin{array}{l}\text { Young's mod- } \\
\text { ulus. }\end{array}$ & Entropy. \\
\hline
\end{tabular}
thermodynamic variables are listed in table 1 .

TABLE 1 .

The analogy may be carried even further in that all three models obey Joule's law, the internal energy being entirely kinetic and independent of the extension, volume or magnetic moment, as the case may be. It can also be shown without difficulty that none of these models permits the use of Boltzmann's principle to enable absolute calculations of entropy to be made. The physical reason for this is obvious; all three models admit of an infinite number of distinguishable configurations unless the chains are completely stretched out, the magnetic material magnetized to saturation, or the gas compressed to zero volume. Differences of entropy can, however, be defined, e. g., in terms of ratios of volume. Some of the more refined models (e. g., van der Waals model of a gas and Weiss model of a magnetic material) which attempt to take interactions between 
atoms into account still possess this same objectionable feature although they do succeed in correlating a large amount of experimental material. As in analogous problems, for which an explicit specification of the entropy itself is required, such as the discussion of vapor pressures, it becomes clear that quantum considerations must be introduced in order to get a model in which the number of distinguishable configurations is finite, and to obtain sensible physical results. It is, however, not difficult to see how such refinements must proceed. We are guided in the first place by analogy with the Brillouin treatment of paramagnetism, in which each atomic magnet is only allowed a finite number of orientations, and we begin by looking for some similar feature in a polymer chain.

For the pure paraffin chain it is possible to state fairly accurately what the model must be like, Pitzer [20], Taylor [28]. For ethane the following model is consistent with both thermodynamic and spectroscopic evidence: the two methyl groups can rotate about the common single bond, but there are three possible energy minima separated by potential barriers of the order of $3 \mathrm{kcal} / \mathrm{mole}$, so that the coordinate $\phi$, measuring the angle of twist, is associated with a contribution to the energy of the form

$$
E=E_{0}(1-\cos 3 \phi) .
$$

It is natural to ask whether an energy function of the form (6) can be applied to each link in longer chain paraffins. If it can, then it becomes plain that the difficulty in assigning a finite entropy to the hydrocarbon chain disappears. For, at a sufficiently low temperature, a chain in equilibrium will be near one of the positions of minimum energy called for by (6). The effect of thermal agitation can then be described accurately enough as leading to a contribution to the potential energy of the order of $E_{0} \sum_{r} \frac{\left(3 \phi_{r}\right)^{2}}{2}$, where $\phi_{r}$ is the "twisting coordinate" associated with the $r^{\text {th }}$ link in the chain. The discussion of the problem is then that of a set of simple harmonic oscillators, which can be carried out exactly on a quantum mechanical basis. If it were correct to discuss a long paraffin chain in this way, it could be concluded that the entropy associated with thermal agitation should approach zero as $T \rightarrow 0$, leaving only the term $k \ln 3$ per link as the maximum possible residual entropy, associated with the fact that, according to an energy-function such as (6), each carbon atom can be linked to the next in three geometrically distinguishable ways. This argument holds equally well even if we take account of degrees of freedom associated with bond lengths as well as bond orientations. For, at a low enough temperature, bond lengths must also be near their equilibrium values, and the effect of departures can also be expressed by means of a quadratic energy function. If we suppose further that these various degrees of freedom are coupled, we should obtain the energy that will contain product terms as well as squares. It is always possible, however, to find a linear transformation of coordinates that reduces such a quadratic form to a sum of squares, at the same time avoiding the introduction of product terms into the kinetic energy. This means that the low temperature behavior of the "stretching", "bending", and "twisting" modes of a hydrocarbon chain is equivalent to that of a set of simple harmonic oscillators, which contributes nothing to the residual entropy.

On this basis it is possible to give a satisfactory qualitative account of the specific heats of polymers. The degrees of freedom of independent chains should be described with reasonable accuracy by a "onedimensional" spectrum of Debye type, containing both transverse and longitudinal modes, which would lead to a specific heat proportional to $T$ at low temperatures. The interactions between chains can be described by adding to this a three-dimensional Debye spectrum, leading to a contribution to the specific heat proportional to $T^{3}$ at low temperatures, and the phenomena of crystallization and the melting of crystals would also be consequences of this interaction. Finally, the "glass-like transition region" marks the range of temperature at which the jumps over the potential barriers occurring in expression (6) become frequent enough for the twisting modes of the chain to come into action. At lower temperatures each chain would be "stiff at the joints" but the stretching modes would remain in operation. In other words, the glass-like transition ought to correspond to the appearance of rubber-like properties and to a rapid rise in the specific beat connecting low and high temperature portions of roughly one-dimensional Debye type, the high temperature part having a lower characteristic Debye temperature. These features seem to agree well with what is observed in practice for the specific heats of various polymers, e. g., Furukawa et al. [6], but only preliminary quantitative analysis on this basis seems to have been carried out. It may well be that too many adjustable constants are involved. The model will not be discussed further from this angle, but the above considerations suggest strongly that it must be a reasonable first approximation.

If an energy function such as (6) were applicable to each link of a very long chain, one could say that the three configurations at any given joint should occur with very nearly equal frequency, and that this leads to a residual entropy of the order of $k$ per "link". (The crude value $k$ in 3 has to be reduced somewhat to allow for steric effects.) Later on we shall assemble some experimental evidence showing that a smaller value is, in fact, more likely to be correct. We are therefore prompted to ask the following two questions:

(a) To what extent is an energy function such as (6) an adequate description of a long chain molecule?

(b) How much is the entropy affected by the deletion of sterically impossible chain configurations?

Since a residual entropy of $k$ per unit would still be noticeable thermochemically, an attempt to set a more precise upper limit is justified. Partial answers to both questions have been obtained by Pitzer [20] and Taylor [28]. From a study of the thermodynamic properties of various hydrocarbons Pitzer con- 
cluded that expression (6) was an oversimplification, and that the trans configuration in which any three successive bonds in the main chain lie in one plane is, energetically, somewhat more favorable than any other one, involving one or more gauche linkages. For brevity we shall hereafter refer to trans configuration of bonds as "straight", and to any departure from it as a "kink", although the straight configuration is the well-known zigzag pattern. Pitzer [20] and Taylor [28] propose to replace expression (6) by something like

$$
E=E_{0}(1-\cos 3 \phi)+E_{1}(1-\cos \phi)
$$

and they estimate that $E_{0}$ and $E_{1}$ should be so chosen that if a straight or trans configuration is taken as having an energy of zero, a kinked or gauche configuration should have a minimum energy of about +0.8 $\mathrm{kcal} / \mathrm{mole}$, and that the maxima separating the three minima should have peaks of about $+4.1 \mathrm{kcal} /$ mole. These figures have been shown to be nearly right by studies of a large number of related hydrocarbons; see, for example, Pitzer [20], [21]; Pitzer and Kilpatrick [23]; Beckett, Pitzer, and Spitzer [1]; Pitzer and Beckett [22]; Person and Pimentel [19]. Confirmation that the steric energy difference is of this order of magnitude for butane, pentane, and hexane is provided by the spectroscopic studies of Sheppard and Szasz [26]. Such a modification in the energy function has some important consequences. It means, in the first place, that the probability that any three successive bonds lie in one plane is not now the simple factor $\frac{1}{3}$ but the significantly larger value $\frac{1}{1+2 e^{-E_{2} / k \mathrm{~T}}}$, where $\mathrm{E}_{2}$ corresponds to the figure of $0.8 \mathrm{kcal} / \mathrm{mole}$ just mentioned. Secondly, such a chain should tend to "straighten out" as it cooled, an effect that is not predicted at all by the simpler model, but for which there is a little experimental evidence. Of course, the glass-like transition intervenes before the stra'ghtening-out is complete, otherwise the straight configuration would be the only one that would have to be considered at all at absolute zero, and the configurational entropy would be zero.

The necessity of working with the function (7) instead of (6) appears to be a consequence of interactions between noncontiguous methyl and $-\mathrm{CH}_{2} \mathrm{C}-$ groups, and it seems necessary to ask whether one is likely to have any success in applying the same type of function to more complicated cases. Two complicating factors are the presence of side substituents and the possible occurrence of double bonds or other types of linkage in the main chain. The former factor is discussed by Flory [5, ch. 6]. His conclusions are, in effect, equivalent to saying that a function such as (7) should still be adequate, but that the chain configuration of lowest energy is, in general, no longer a simple zigzag. Steric considerations imply that some other more complicated configuration may have to be chosen as the "reference" one, departures from which determine the energy as given by function (7). For the present purposes this is not serious, but it does have to be borne in mind if one is attempting to compute e. g., the effective "spread" of a chain. In the extreme case of polyisobutylene only one configuration could be found that seemed to be sterically possible at all. In cases like this, the energy differences occurring in (7) would presumably be greater than they are for paraffins with a correspondingly smaller entropy at any given temperature. On the other hand, the higher potential barriers would also tend to lead to a higher glass-like transition temperature, so that it does not follow that introducing side substituents will automatically lower the residual entropy. Very little seems to be known about the theoretical effect of double bonds in the main chain, though it is generally considered that they should facilitate rotations of single bonds adjacent to them.

The effect of introducing a triple bond has been examined by Osborne, Garner, and Yost [18] who studied the thermodynamic properties of dimethylacetylene $\mathrm{CH}_{3}-\mathrm{C} \equiv \mathrm{C}-\mathrm{CH}_{3}$. They concluded that the potential barriers restricting relative rotation of the methyl groups were probably not higher than $0.5 \mathrm{kcal} / \mathrm{mole}$, and gave reasons for thinking that the main effect of the triple bond is simply that the methyl groups are much further apart than they are in ethane. They argue that a double bond ought to have similar consequences in reducing the height of the barrier. The barrier in olefin hydrocarbons for groups adjacent to the double bond is about 1 to $2 \mathrm{kcal} / \mathrm{mole}$. See Kilpatrick and Pitzer [13], who studied ethylene, propylene, and the butenes. This is significantly smaller than for paraffins, but there does not seem to be any information available about the existence or nonexistence of an effect analogous to the energy difference between trans and gauche configurations that were noted for paraffins. The indirect experimental evidence is certainly consistent with the idea that, in rubberlike polymers, the potential barriers between different chain configurations are lower than in paraffins, and that the bias in favor of straight configurations may be small or nonexistent. If we could neglect other steric effects such as the fact that a chain may not cross itself our present problem would be solved. We could say that, as the relative probabilities of a given $\mathrm{C}-\mathrm{C}$ bond corresponding to a straight or kinked configuration should be in the ratio $1: 2 e^{-E_{2} / T k}$, the partition function must contain a factor $\left(1+2 e^{-E_{2} / k T}\right)$ per link, the corresponding entropy per link being

$$
k\left[\ln \left(1+2 e^{-E_{2} / k T}\right)+\frac{E_{2}}{k T} \cdot \frac{2}{e^{E_{2} / k T}+2}\right],
$$

which falls steadily from $k \ln 3$ to zero as $E_{2} / k T$ rises from zero to infinity. For the discussion of any particular type of polymer chain, it would only be necessary to insert the appropriate values of $\dot{E}_{2}$ and of the glass-like transition temperature into (8) in order to arrive at the expected residual entropy.

We must now examine the effect of removing from the partition function terms corresponding to self- 
crossing configurations which are implicitly included in the calculations leading to (8). Such problems are of considerable mathematical and physical interest, and although only a very few highly idealized ones can be solved analytically (for example, we do not yet know the equation of state of a perfect gas of rigid spheres), it seems possible to get an answer that is sufficiently good for present purposes. It is known [7,8] that the problem of an actual chain cannot be treated merely as a small perturbation of the "unrestricted" random walk problem. The effect of introducing any realistic type of constraint always results in a significant reduction in the entropy per link. Stated otherwise, the number of possible configurations of the restricted chain is only a very small fraction of that for the unrestricted one. Some writers have introduced the concept of the "equivalent random walk". They suppose that for sufficiently large $N$, the behavior of a real chain $N$ links long is analytically equivalent to that of a random walk of $N / n$ steps, with a suitable choice of $n$ and of the length of step. Unfortunately, recent work, Wall et al. [32, 33], Temperley [29] indicates that this concept is not strictly valid in two dimensions, nor in three dimensions for values of $N$ small enough to be of physical interest, at least not if we wish to treat $n$ as independent of $N$. Nevertheless, the concept is of value and will be used again later. If, for example, we had to do with an extremely rigid chain, strongly biased in favor of straight configurations, it would be possible to define some number, $m$, that measures the average distance between two kinks. We should expect this to be of the same order as the $n$ just introduced, though the concepts of length of step of equivalent random walk and length of link of equivalent flexible chain are logically distinct.

A start was made on the steric problem by Taylor [28] and a very similar process was used by Montroll [16] for the idealized case in which the links of the chain are constrained to lie along a plane square lattice. These authors show that the effect of removing from the partition function terms corresponding to self-crossing paths of a certain type can be established rigorously and very simply. The type of path deleted is, for the plane square lattice, any path containing one or more closed loops consisting of single squares, for example, a path passing in succession through the points $(1,2),(2,2),(2,1),(1,1)$, $(1,2)$. Paths containing longer loops are retained for the present. We summarize Montroll's argument for the plane square lattice, each link being constrained to be at right angles to those that precede and follow it. The first link can take any one of four directions. We can then proceed to the next link by means of either a left or a right turn, which we shall denote symbolically by $E$ or $F$. The passage from the second to the third link also involves either a left or right turn, and so on. If we retained self-crossing paths, our generating function for a path $n+1$ links long would be simply

$$
4(E+F)^{n}
$$

each term in this product, with the factors $E$ and $F$ written in their proper order, corresponding to a possible path. We shall now show how expression (9) is modified if we delete terms containing either of the successions EEE or $F F F$, as either of these combinations corresponds to the enclosure of a single square. By induction, we can determine the generating function $S_{n}$ that replaces (9) for a path $n$ units long if we know it for all shorter paths. Let $S_{n}(E)$ and $S_{n}(F)$ be those parts of $S_{n}$ whose final factors are $E$ or $F$ respectively. Now $S_{n}(E)$ may end in one of the two ways $F E$ or $F E E$, but in no others, since three $E$ 's in a row are forbidden. Thus we have the relation

$$
S_{n}(E)=S_{n-1}(F) \cdot E+S_{n-2}(F) \cdot E \cdot E
$$

and a similar one for $S_{n}(F)$. If we are concerned, as we are here, simply with the counting of configurations in which left and right turns are equally weighted, $E$ and $F$ can be replaced in equations such as (9) and (10) by a single variable $x$, so we find that $S_{n}(x)$ is determined by the difference equation

$$
S_{n}(x)=x S_{n-1}(x)+x^{2} S_{n-2}(x)
$$

which can be solved by a process quite analogous to that for linear differential equations, that is, by looking for solutions of the type $S_{n}=A p^{n}$, where $A$ and $p$ are independent of $n$, but may depend on $x$. Such a solution will satisfy (11) provided that

$$
p^{2}-x p-x^{2}=0 \text { or } \frac{p_{1}}{p_{2}}=x\left(\frac{1 \pm \sqrt{5}}{2}\right)
$$

giving us two independent solutions, each of which can be multiplied by an arbitrary function of $x$ and the two can then be added. It is readily verified that this is the most general solution possible, and the two arbitrary functions are completely determined from the known forms of $S_{1}$ and $S_{2}$. For the purpose of determining the entropy of a chain $n$ steps long, as $n$ becomes large, the answer can be read off directly from (12), because the complete solution is $A_{1} p_{1}{ }^{n}+A_{2} p_{2}{ }^{n}=S_{n}$ and the term involving $p_{2}{ }^{n}$ can contribute only an insignificant amount to the entropy compared with that in $p_{1}{ }^{n}$. We have, in fact, the result that the entropy per link is reduced from the value $k \ln 2$ that would follow from (9) to the distinctly smaller value $k \ln \left(\frac{\sqrt{5}+1}{2}\right)$, as a result of deleting a certain type of impossible path. This method can readily be generalized to other types of lattice. For example, Taylor [28] showed that the removal of "pentane configurations" reduced the configurational entropy of a linear paraffin from the value $k$ ln 3 per link that we calculate on the basis that successive links must be inclined at the tetrahedral angle to the value $k \ln (1+\sqrt{2})$. His result is equivalent to replacing equation (11) by

$$
p^{2}-2 p x-x^{2}=0 \text { or } p=x(1 \pm \sqrt{2}) \text {. }
$$


By pentane configuration is meant any configuration that nearly closes upon itself in four successive steps, which cannot occur for any paraffin shorter than pentane. The fact that in such cases sizable reduction in the entropy occurs when we remove a particular type of sterically impossible path enables us to understand the failure of past attempts to treat the effect of steric obstruction simply as a small perturbation of the random walk problem. For a long chain, any significant reduction in the entropy per link means that only a very small fraction of the random walks gives permissible chain configurations.

There is no particular difficulty in extending the above work to other types of lattice, including cases in which certain configurations of successive bonds are, a priori, more probable than others. Thus, in Montroll's discussion of the square lattice [16] two adjacent links were not permitted to be in the same straight line, but it is quite possible to extend the discussion to a more general case in which rightangles and straights have different initial probabilities. It becomes much more difficult if we now attempt to remove paths involving longer loops. This question has been studied by the writer elsewhere [29] for the plane square lattice by methods differing from those outlined above, and, while the problem of writing down the generating function for all noncrossing paths is still unsolved, it is believed that enough is known about the properties, of this function to enable the entropy to be determined. We quote the result here:

$$
S=k\left[\ln \left(1+\sqrt{2} e^{-E_{2} / k T}\right)+\frac{E_{2}}{k T} \cdot \frac{\sqrt{2}}{e^{E_{2} / k T}+\sqrt{2}}\right],
$$

which, it will be observed, is of precisely the form (7) with 2 replaced by $\sqrt{2}$. $\quad E_{2}$ here has the meaning of the gain in energy involved in replacing a right-angle between two successive links by a straight. If we put $E_{2}=0$ and compare (13) with (12) we arrive at the remarkable conclusion that removing just pentane configurations from random paths, the links inclined at the tetrahedral angle, has precisely the same numerical effect on the entropy as has removing loops of all sizes from random paths disposed along the square lattice, the steric effects being at least as severe in the paraffin chain as they are in the square lattice one. A removal of loops larger than those corresponding to pentane configurations will certainly diminish the paraffin entropy further. We wish to take account of the effect of a finite $E_{2}$, as well as of steric effects, in the paraffin chain, and it seems safe to regard (13) as an upper limit for these purposes. With the value of $E_{2}$ of $800 \mathrm{cal} / \mathrm{mole}$ and a glass transition temperature of the order of $200^{\circ} \mathrm{K}$, we should have $e^{E_{2} / k T} \approx 7$, so that we estimate $0.5 k$ per link as an upper limit for the configurational entropy of a paraffin-like chain. The corresponding length of effective segment is six $\mathrm{C}-\mathrm{C}$ links. For a rubber, $E_{2}$ may be negligible, and our estimate becomes about $k$ per link for the upper limit, and the corresponding effective segment is 1.7 monomer units.
There is still one factor, not negligible, to consider, but there are no accurate means of estimating its numerical importance; this is that the occurrence of crystallization in polymers (in some cases up to high temperatures) shows that the interaction between chains is not negligible, and it can be foreseen that any steric effects between neighboring chains will have the effect of lowering the configurational entropy still further. This is probably true in a liquid paraffin as well as in a solid. If this effect is significant it would mean that the entropies actually occurring would be appreciably below the above estimates, and there is experimental evidence that this is sometimes the case. Various independent attempts to estimate the length of the effective segment give values for this quantity running appreciably higher than those that would be calculated for an isolated chain by the method outlined above. In this way the influence of the neglected factor of interaction between chains seems to be about comparable with that of interaction between distant parts of the same chain. There is some evidence that this neglected factor has a stronger influence in solids than it has in liquids or solutions, and this is to be expected.

\subsection{Analysis of Certain Experimental Results}

The preceding theoretical considerations suggest that the configurational entropy of a polymer chain may be significantly below the value $k \log 3$ per link that one estimates from "elementary" considerations. The two possible causes for this are: (a) The intrinsic properties of the linkages themselves, e. g., straight configurations may be favored by the nature of the interactions between neighboring links, as in the paraffin chain. (b) Steric effects tend to reduce the number of "coiled-up" configurations. This includes both interactions between neighboring chains and between distant parts of the same chain, while (a) is the result of short-range interactions within chains. What we shall try to estimate from experimental data is the effective length of segment or "average length of chain between kinks." For the paraffin chain, all the $\mathrm{C}-\mathrm{C}$ bonds in such a segment lie in one plane and assume the zigzag pattern. For other types of chains the geometry of the configuration of lowest energy may be more complicated, but we shall still refer to it as straight, to a departure from it as a kink, and to the mean distance between two kinks as an effective segment. Strictly speaking, the last concept is logically not quite equivalent to that of length of step of equivalent random walk but the ratio of the two is not likely to be far from unity. It is, in principle, possible to distinguish experimentally whether (b) is the dominant consideration or whether (a) is also significant. In the latter case, the effective length of segment should increase as the temperature falls and kinks become less likely, and there does seem to be some evidence that this occurs.

The evidence may be roughly divided as follows, one subsection devoted to each topic: 
1. Studies on solid polymers. Apart from the thermodynamic properties already referred to, as being in reasonable accord with the model we are using, there is a little further evidence giving direct information on the segment length in a few cases.

2. Studies on polymer solutions. The information here is mainly indirect, and is usually equivalent to studying the effective size of the coiled up polymer molecule of known molecular weight. For a completely flexible chain, the effective linear dimension of a coiled up molecule is proportional to $N^{1 / 2} l$, where $N$ is the number of links of end-to-end length $l$. If straight configurations are so much favored that there is on an average only one kink per $m$ links, such a chain should behave, to a first approximation, like a flexible one of $\frac{N}{m}$ links, each of length $A m$, (where $A$ is a constant, of the order of unity, depending on the geometry of the straight configuration) and its mean dimension should be increased by a factor $A m^{1 / 2}$. We can deduce the value of $m$ by comparing the dimensions of the molecule, as inferred from experiment, with that calculated from bond lengths and angles on a random walk basis. Effects (a) and (b) both tend to increase $m$, and it is only possible to separate them in a few cases.

3. Studies on solid and liquid paraffins and related compounds. There is quite a variety of evidence pointing to the conclusion that such molecules, (up to about 30 carbon atoms), behave roughly like nearly rigid rods. Such a conclusion is in good agreement with the thermodynamic properties of the paraffin chain discussed above.

\section{a. Studies on Solid Polymers}

It has been noted (and perhaps this is the most suggestive point) that the tensile strength of a typical polymer remains practically zero until a degree of polymerization of about 50 is reached, after which it begins to increase rather suddenly. It is easy to assume that the real distinction between a "wax" and a "fiber" is that the molecules of a fiber cannot be sheared over one another indefinitely because the kinks become entangled while the molecules of paraffin wax are nearly straight and can slide smoothly past one another. One arrives at an effective distance between kinks of the order of 15 to 20 units, on the basis of the rather crude assumption that molecules would have to have at least two kinks before they could serve as "double-hook" units, which could theoretically form a structure capable of resisting tension.

The fact that above about a two-fold extension, the tension in rubber rises much more rapidly with the extension than the simple "random-chain" theory predicts, indicates that the maximum obtainable extensions, (of the order of 5 to 10 fold) really do represent a situation in which an appreciable fraction of the chains are pulled into configurations approaching maximum extension. This conclusion holds equally well whether, with Treloar [30], we regard the observed stress-strain curves as quali- tatively explained by James and Guth's [11] treatment of highly extended chains, which does predict just such a rapid increase in elastic modulus as full extension is approached, or whether, with Flory, [4] we attribute this increase in elastic modulus mainly to the effect of crystallization. Crystallization is facilitated by stretching out the chains thus making the configurations of neighboring chains more nearly alike. If a 5 to 10 fold extension is really the maximum that can be obtained without rupture of an appreciable fraction of the chains, this would mean that

$$
\left(\frac{N}{m}\right) \cdot m \approx 5 \text { to } 10\left(\frac{N}{m}\right)^{1 / 2} \cdot m
$$

or $\frac{N}{m} \approx 30$ to 100 , whereas, in an actual lightly vulcanized rubber, the distances between cross-linkages probably correspond to chains at least several hundred units long. Thus it seems difficult to reconcile the observed elasticity of rubber with a value of $m=1$, but postulating a value of the order of 2 to 5 would improve matters considerably. Treloar [30, ch. 8] concluded, on the basis of studies of the double refraction of solid rubber under stress, that $m \approx 2$, the precise numerical value depending on the exact way in which we define $m$.

A very recent study, Honnold, McCaffrey, and Mrowca [10] has been made using proton resonance methods. It is difficult to obtain actual estimates of segment length directly by this means, but their studies of silicones, and their comparison of polypropylene and polypropylene oxide do indicate that the introduction of oxygen linkages into the main chain makes it more flexible. This is in agreement with the conclusions of Kuhn and Kuhn, [14] obtained from the birefringence of solutions.

\section{b. Analysis of Data on Solutions of Polymers}

There are two basic ways to determine the length of the effective segment. The most direct is to treat the large polymer molecule as if it were an aggregate of effective segments, each free to move short distances in the solution, and to assume various orientations. These changes in the position and orientation of the segments correspond to movement of the kinks up and down the long polymer molecules, the average number of kinks remaining constant. This movement of segments as a result of thermal agitation will resemble that of a solute molecule in an ordinary solution, except that the segment is not free to wander very far from the center of gravity of the polymer molecule. This situation is described by the so-called lattice model of a polymer solution, as amended by Flory to cover the case of extremely dilute solutions. See Flory [5, ch. 12] for a summary. Using this treatment, we can estimate the size of the effective segment in several ways.

(a) From the osmotic pressure. Since this is an equilibrium property, we can use solution theory directly. The limitation is set by the availability of data. The solutions have to be very dilute for 
existing theory to be acceptable, whereupon the pressures to be measured become rather low for accurate measurement.

(b) From the viscosity. The idea here is that any motion of a segment through the solvent is associated with the displacement of an equivalent volume of solvent molecules. These molecules have to be removed from the liquid, leaving a "hole" big enough to contain the segment. The segment then enters this hole and the space it leaves vacant in the liquid is filled by the displaced molecules. Independently of any particular model of the liquid, we can argue that the energy barrier associated with such a process must be of the order of magnitude $l \rho v$, where $l$ is the latent heat of evaporation; $\rho$ is the density of the solvent and $v$ the volume of the effective segment. Thus the probability of such a process may be expected to be proportional to $\exp (-l \rho v / k T)$, and the viscosity of the solution should contain a factor that is the inverse of this. If so, the activation energy $l \rho v$ can be estimated by means of the customary plot of $\log$ (viscosity) against $1 / T$. For a survey of the conclusions from these two methods we quote Powell and Eyring [25, p. 191]. "It is worth noting that the segment lengths estimated from osmotic pressure data (18 to 33 atoms for polystyrene and polyethylene oxide) and from melting point data ( 20 to 25 atoms for hydrocarbons) are in agreement with the segment lengths from viscosity data (20 to 25 atoms for hydrocarbons, 28 to 34 atoms for polyesters)." The reader is referred to this review article for a survey of the work leading to these conclusions.

Eyring and his collaborators have attempted an alternative estimate of segment length from viscosity data, based on a viscosity formula of the type

$$
\eta=A \exp (u / k T)
$$

in which attempts are made to estimate $A$, as well as $u$, from the experimental results, and to compute a theoretical value of $A$ in terms of segment length. In practice, this method fails to give useful results, but this failure does not mean that the estimates of $u$ are invalid also. Suppose, for example, that $u$ is varying slightly with temperature, say according to the law $u=u_{0}(1+\alpha T)$. The customary logarithmic plot of the viscosity data against $1 / T$ would now yield $u_{0}$ and $A e^{\alpha u_{0} / k}$ in place of $u$ and $A$, and a value of $\alpha$ of no more than a few tenths of one percent per degree could lead to a completely false experimental estimate of $A$. A similar difficulty occurs in the analysis of thermionic data in metals.

(c) A third method of estimating segment lengths, due to Kuhn and Kuhn [14], rests on the study of the double refraction of a polymer solution that is being steadily sheared, the shearing flow tending to orient the typical segment and stretch out the polymer molecule in a manner that depends essentially on the length of the typical segment. By a rather complicated method of analysis, these authors arrive at results ranging from segment lengths of the order of 30 carbon-carbon links for paraffin-like polymers ranging down to 1 to 3 monomer units for rubber, hydrogenated rubber, and polymers which contain $\mathrm{C}-\mathrm{O}$ linkages in the main chain. Such linkages, by increasing the spatial separation of the $-\mathrm{CH}_{2} \mathrm{C}-$ groups, no doubt make the chain more flexible, as already suggested above.

The second basic method of attack on this question is based on an attempt to determine the linear dimensions of the molecules in solution, and to examine how these differ from the values to be expected on the completely random-walk basis. This should give a measure of $m$, since we have pointed out that the ratio, actual dimension to calculated dimension, should be approximately equal to $m^{1 / 2}$. The actual dimension is obtained by an ingenious analysis of data on the viscosity of dilute solutions, due to Flory [5, ch. 14]. This new method of analysis of viscosity bears no relation to the method we have already described, but is not inconsistent with it.

The very dilute polymer solution can be regarded as a mixture of roughly spherical aggregates representing the molecules of polymer, separated by fairly large regions of pure solvent. Flory's idea is to suppose each molecule of polymer replaced by an equivalent solid sphere, and to attribute the excess of the viscosity of the solution over that of the pure solvent to the fact that the solvent cannot flow freely through the polymer molecules, but is partly diverted around them. One can then apply Einstein's wellknown treatment of the viscosity of a liquid containing a random distribution of solid spheres. Thus, according to this approach, we consider that a whole polymer molecule obstructs the flow of the solvent to an extent that depends on its spread in space, and the motion of individual segments is not considered.

Flory shows that, if the temperature is properly chosen in relation to the solvent and concentration, the spatial distribution of polymer molecules in the solution is indeed random; and further, that the extension of the molecule in space is then very nearly the same as what we should calculate on the assumption that the interactions of solvent molecules and polymer segments with one another and among themselves are equivalent. At this temperature, called by Flory the $\theta$ point, the solvent becomes, so to speak, "neutral", any actual differences in these interaction energies being approximately balanced by the effect of thermal agitation. Various other definitions of the $\theta$ point are possible; for example, it can be shown that it should correspond to the limit of solubility of a sample of polymer of extremely high molecular weight. A solution at its $\theta$ point should then contain a practically random spatial distribution of molecules in the solvent, and the average chain configuration of these molecules should be that which we should predict on the basis of neglect of long-range interactions between segments (apart from steric effects).

Flory's analysis [5, ch. 14, p. 618] leads him to conclude that "the observed end-to-end distances, unperturbed by long-range interactions, are consistently greater than would hold if bond rotation were completely unrestricted. Steric effects of one sort or another probably are responsible." The 
factor measuring the "expansion of linear dimensions", which should be a measure of $m^{1 / 2}$, ranges from 1.60 for gutta-percha to 4.9 for cellulose tributyrate, in satisfactory qualitative agreement with figures for $m$ already calculated for paraffin-like and rubber-like polymers by other means.

It is of some interest to ask what is likely to be the relative importance in causing this expansion of the molecule of the two distinct factors met in discussing the hydrocarbon chain, the fact that two carbon atoms may not be in the same place, and the fact that there may be correlation between the directions of e. g., neighboring $\mathrm{C}-\mathrm{C}$ bonds. The first of these factors should lead to an expansion that remains the same at all temperatures, while the second should lead to an expansion that increases as the temperature falls. Calculations on a paraffin-like chain, Taylor [28], Kuhn [15] indicate that the endto-end length should increase by a factor of 1.2 as the temperature falls from $+100^{\circ} \mathrm{C}$ to $0^{\circ} \mathrm{C}$. Flory [5] finds evidence of just such an effect for various solutions of cellulose tributyrate, each one studied at its $\theta$ point. This is also the very molecule that shows the biggest departure from the random-walk configuration.

On the basis of all this evidence, we seem justified in concluding that:

a. The absence of configurations in which two carbon atoms coincide increases the linear dimensions by a factor of the order of 1 to 5 above what they would be on a random-walk treatment. This effect seems to be the only important one in rubberlike materials. It implies that only about half the linkages between isoprene units can be regarded as uncorrelated with one another, and leads to a residual entropy of $1 / 2 k \ln 3$ per isoprene unit.

b. For a paraffin-like chain there is often an additional effect, due to correlations between neighboring links probably attributable to interactions between the $\mathrm{CH}_{2}-\mathrm{C}$ groups in the main chain. This seems to be capable of raising the effective value of $m$ from a value of the order of 2 that seems to follow on the basis of factor (a) alone to a value of the order of 20 or more. The corresponding residual entropy is then of the order of 0.1 to $0.2 k$ per monomer molecule. It would not be correct to quote a figure as low as $0.05 k$ corresponding to an effective segment 20 units long, because of the presence of the second term in eq (8).

These values agree remarkably well with those based on the purely theoretical study, bearing in mind that one factor, the interaction of the chains in a solid with one another, was not considered. It is satisfactory that the apparent values of the length of the effective segment are, for both rubber and the paraffin-like polymers, a factor of 2 or so greater than we estimate theoretically for an isolated chain. These experimental studies are almost all based on. dubious assumptions, or depend on numerical constants that have been. evaluated very tentatively. Any one of them, taken alone, would not be very impressive, as comparatively small errors in some of the numerical constants might make a considerable difference to the calculated values of $m$. The results of a number of quite independent investigations all give calculated values of the same order of magnitude, and their cumulative value as evidence is very considerable.

We shall close with a survey of the properties of liquid and solid paraffins and similar bodies of chain lengths between about 10 and 60, which seems to confirm the above conclusions obtained directly from polymer studies.

\section{c. Analysis of Data on Paraffins and Allied Series}

Convincing evidence that paraffin molecules up to about 40 units long pack like rods in the solid state is provided by the X-ray work of $\mathrm{A}$. Müller. For an account of his work the reader is referred to a review article by Daniel [3]. In addition to determining the structure of the ordinary solids, Müller was able to elucidate the nature of a transition that occurs a little below the melting point for paraffins of lengths between 16 and $32 \mathrm{C}$ atoms. He was able to show that this transition is associated with a change in crystal structure, the packing changing from a type natural for flat ribbons to one appropriate to practically symmetric round rods.

The following evidence that in the high temperature state the molecules really are rotating as rigid rods rather than twisting is available. In the first place, the entropy change associated with the transition has been found to be practically independent of chain length, which is what we should expect if the entropy change is mainly associated with a change in the two-dimensional packing of rigid rods. But if only short segments of the chain were rotating at any one time and some of the bonds were being twisted, one would expect the entropy of transition to be proportional to the number of segments, and thus to the length of the chain. Also, the transition temperature is a linear function of the chain length $y$, of the form $A+B y$. This, combined with the result that the entropy of the transition is practically independent of chain length, implies that the energy change associated with the transition must be a similar linear function. This is just what would be expected if the chains are behaving as rigid rods, one contribution to the energy coming from the interaction between any chain and those lying alongside it, which should be proportional to the length of the chain, $y$. A second contribution might be expected from the interactions of methyl groups belonging to end-to-end neighbors, which should be practically independent of chain length.

This type of transition occurs only over a limited range of chain lengths simply because, at the top and bottom of this range, the paraffin melts before undergoing the transition. The melting temperatures of various homologous series are well represented by the empirical relation

$$
T_{m}=(C+D y) /(E+F y)
$$

in contrast with the linear relation for the transition temperature just mentioned. It is now natural to ask whether eq (14) is also to be regarded as evidence 
that paraffin chains are behaving as practically rigid rods. In view of the tentative nature of present theories of liquid structure, any attempt to be dogmatic on this point would be out of place. It does, however, seem worth while to mention two possible interpretations of (14), which, although incompatible with one another, are both in agreement with the picture of a fairly long effective segment. It could be argued that since for all homologous aliphatic series, a relation such as (14) seems to hold, and that the melting temperature settles down to a practically constant value for $m \approx 30$, that this represents the length of the effective segment; that lower hydrocarbons are behaving like rigid rods in both solid and liquid states, while higher ones are behaving as if they were composed of effective segments of about 20 to 30 links long, each of which is capable of moving around in the liquid as a unit, corresponding to the motion of individual kinks up and down the chains. We have already met this picture in discussing polymer solutions. On the other hand, an interpretation of relation (14) can be given on the basis that the chains behave like nearly rigid rods for all values of $y$ for which reasonably pure preparations can be made. The energy change associated with melting should be the sum of interactions between side-toside and end-to-end neighboring chains, and this should be a linear function of $y$, as before. In the liquid near its freezing point it may be supposed that the chains are still packing in a roughly twodimensional fashion, but somewhat more loosely than in the solid, and that each chain also acquires some freedom to move lengthways as well as sideways. Such a picture leads to an entropy change consisting of a constant term, associated with the possibility of sideways movement and rotation, that we have already met in the solid-solid transition, with the addition of a term, roughly proportional to chain length, associated with a possible lengthways sliding of the chains, which would be absent in the solid transition. Such behavior of the entropy and energy of transition leads directly to a relation like (14). For a review of the experimental material on which these considerations are based, and also for some extremely interesting material on esters, which is also in reasonable accord with the "rigid-rod" picture, the reader is referred to Daniel's review article [3]. Simanouti and Mizushima, [27] from a spectroscopic study of liquid cetane $\left(\mathrm{C}_{17} \mathrm{H}_{36}\right)$, concluded that this liquid was probably a mixture of the various possible forms of molecule containing just one kink or gauche configuration, all the remaining linkages being in the trans configuration but the molecules in the solid being entirely of the all trans (coplanar) type. Thus, the effect of the interactions between neighboring molecules seems to be somewhat stronger in the solid, a result that would be expected and this is also in keeping with the idea of an effective segment of $\sim 20$ units.

We conclude by a brief reference to the work of Moore, Gibbs, and Eyring [17], who succeed in interpreting the temperature dependence of the viscosity of liquid paraffins on this same basis of approximately two-dimensional packing, so that the flow of such a liquid resembles the distortion of a crystal under stress by the sliding of layers of molecules over one another, rather than the independent motion of spherical molecules characteristic of ordinary liquids. The latter is what we should expect of the paraffins if there were more than 2 or 3 kinks per molecule, because then they would approximate to spheres far better than to rods.

\section{Discussion and Conclusions}

This enquiry has led into a variety of different fields and provided us with evidence on the chain configuration problem of very varying degrees of relevance and probable reliability, but the over-all consistency of the experimental evidence is surprisingly good, as is the consistency with the theoretical expectations. We have already met a number of cases in which the same data might be interpreted in more than one way, and, in the present state of our knowledge, both ways may well be wrong. Some of the investigations involve numerical constants evaluated by dubious methods, but, for the purposes of the present enquiry, the fact that these investigations were carried out quite independently of one another is a powerful argument in favor of the qualitative conclusions. It is right to mention at this point a few apparent inconsistencies which cannot be explained away so easily. The general picture is that a paraffin-like chain is fairly rigid, and that the introduction of substituents only tends to increase the rigidity, hence a residual entropy of about $0.2 \mathrm{k}$ per link, but that the introduction of a double bond or a $\mathrm{C}$ - O linkage into the main chain should introduce flexibility, and thus put up the residual entropy to as much as 0.5 to $1.0 \mathrm{k}$ per link. And yet, (a) according to theory, and also according to Kuhn and Kuhn [14] and Honnold, McCaffrey, and Mrowea [10] the polyethylene oxide chain should be very flexible, whereas the behavior of this solid is not rubber-like, and osmotic pressure data, Powell and Eyring [25], indicate an effective segment of the order of 20 to 30 atoms. (b) Kuhn and Kuhn [14] studied a hydrogenated rubber, in which all the double bonds were saturated. This should behave like a hydrocarbon chain with one substituent methyl group per isoprene unit, and should therefore form a stiff chain, whereas, in solution, it appears to be at least as flexible as ordinary rubber.

\section{Possible Further Work}

The main conclusions seem confirmed by a number of independent pieces of evidence, but the investigation does suggest possible further lines of enquiry.

(a) The idea of a possible numerical relationship between effective length of segment and fraction of material crystallized seems likely to throw light on the crystallization of rubbers, if it could be made more precise.

(b) It might be worth studying nonsaturated hydrocarbon liquids by $\mathrm{X}$-ray and viscosity methods in order to see whether such molecules pack as approximate spheres, as they should if they contain a number 
of fairly flexible bonds, or whether they pack as long rods. One liquid that might be interesting to study in these ways is squalene, since it consists of six isoprene units, and the average molecule should contain 2 or 3 kinks.

\section{References}

[1] C. W. Beckett, K. S. Pitzer, and R. Spitzer, J. Am. Chem. Soc. 69, 2488 (1947).

[2] R. F. Boyer and E. Spencer, Advances in Colloid Sci. I, 183 (1946).

[3] V. Daniel, Advances in physies, Phil. Mag. Supplement 2, 450 (1953).

[4] P. J. Flory, Ind. Eng. Chem. 38, 417 (1946).

[5] P. J. Flory, Principles of polymer chemistry (Cornell University Press, Ithaca, New York, 1953).

[6] George T. Furukawa, Robert E. McCoskey, and Gerard J. King, J. Research NBS 49, 273 (1952) RP2364.

[7] T. B. Grimley, J. Chem. Phys. 21, 185 (1953)

[8] T. B. Grimley, J. Chem. Phys. 22, 1134 (1954).

[9] E. Guth, H. M. James, and H. Mark, Advances in Colloid Sci. II, 253 (1946).

[10] V. R. Honnold, F. MeCaffrey, and B. A. Mrowca, J. App. Phys. 25, 1219 (1954).

[11] H. M. James and E. Guth, J. Chem. Phys. 11, 455, 531 (1943).

[12] W. Kauzmann, Chem. Rev. 43, 219 (1948).

[13] J. E. Kilpatrick and K. S. Pitzer, J. Research NES 37, 163 (1946) RP1738.

[14] W. Kuhn and H. Kuhn, Helv. Chim. Acta 26, 1394 (1943)
[15] H. Kuhn, J. Chem. Phys. 15, 843 (1947).

[16] E. W. Montroll, J. Chem. Phys. 18, 734 (1950).

[17] R. J. Moore, P. Gibbs, and H. Eyring, J. Phys. Chem. 57, 172 (1953).

[18] D. W. Osborne, C. S. Garner, and D. M. Yost, J. Chem. Phys. 8, 131 (1940).

[19] W. B. Person and G. C. Pimentel, J. Am. Chem. Soc. 75, 532 (1953).

[20] K. S. Pitzer, J. Chem. Phys, 8, 711 (1940).

[21] K. S. Pitzer, Ind. Eng. Chem. 36, 829 (1944).

[22] K. S. Pitzer and C. W. Beckett, J. Am. Chem. Soc. 69, 977 (1947).

[23] K. S. Pitzer and J. E. Kilpatrick, Chem. Rev. 39, 435 (1946).

[24] G. Polya, Acta Math. 68, 145 (1937).

[25] R. E. Powell and H. Eyring, Advances in Colloid Sci. I, $183(1942)$.

[26] N. Sheppard and G. J. Szasz, J. Chem. Phys. 17, 86 (1949).

[27] T. S. Simanouti and S. Mizushima, Sci. Papers Inst. Phys. Chem. Research (Tokyo) 40, 467 (1943).

[28] W. J. Taylor, J. Chem. Phys. 16, 257 (1948).

[29] H. N. V. Temperley (to be published in Phys. Rev.).

[30] L. R. G. Treloar, The physics of rubber elasticity (Oxford University Press, 114 5th Ave., New York, N. Y., 1949)

[31] F. T. Wall, L. A. Hiller, and D. J. Wheeler, J. Chem. Phys. 22, 1036 (1954).

[32] F. T. Wall, L. A. Hiller, and W. F. Atchison, J. Chem. Phys. 23, 913 (1955).

[33] F. T. Wall, L. A. Hiller, and W. F. Atchison (unpublished data).

Washington, April 18, 1955. 